\title{
Images in pediatrics: The thymic sail sign and thymic wave sign
}

\author{
Nuno D. Alves • Marta Sousa
}

Received: 9 October 2012 / Accepted: 15 October 2012 / Published online: 30 October 2012

(C) Springer-Verlag Berlin Heidelberg 2012

\begin{abstract}
The authors present a radiographic image portraying the "thymic sail sign" and the "thymic wave sign," both normal findings in infant radiographs and present a short description of these signs. These are distinguished from pathologic findings such as the "spinnaker-sail sign" in pneumomediastinum.
\end{abstract}

Keywords Thymus gland · Thoracic radiography

A chest radiograph obtained for a 7-month-old female baby with respiratory infection showed normally aerated lungs and a bulging thymic lobe over the right hemithorax (white arrow). This is the classic "thymic sail sign" [2], a frequently encountered radiographic finding in pediatrics, representing the triangular extension of the normal thymus out laterally in children's chest radiographs (Fig. 1). The thymic contour is convex and the inferior border is straight, giving it a sail-like appearance. It is important to recognize that pneumonia of any lobe would not present as a mediastinum-based opacity with a horizontal inferior border.

The thymic sail sign is a normal finding in infants and should not be confused with the "spinnaker-sail sign" where the thymic lobe shadow is laterally and superiorly displaced due to pneumomediastinum, as though an actual boat sail blown by strong wind [1]

N. D. Alves $(\bowtie) \cdot M$. Sousa

Hospital do Espírito Santo de Évora EPE,

R. Julio Dinis 931 4D,

4050-327 Porto, Portugal

e-mail: nuno.dalves@hotmail.com

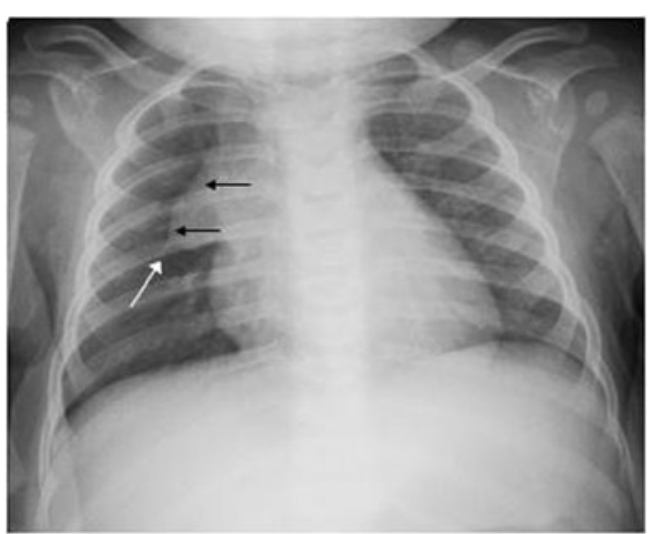

Fig. 1 The classic thymic sail sign (white arrow) and thymic wave sign (black arrows)

The normal thymus has a soft texture and therefore may on radiographs present an undulation of its contour caused by the ribs, known as the "thymic wave sign" (black arrows) [2]. This appearance helps to exclude mediastinal expansive masses such as teratomas. These would not have a horizontal inferior border and may present with calcifications, whereas the normal thymus has soft tissue density.

\section{References}

1. Bejvan SM, Godwin JD (1996) Pneumomediastinum: old signs and new signs. AJR Am J Roentgenol 166(5):1041-1048

2. Nasseri F, Eftekhari F (2010) Clinical and radiologic review of the normal and abnormal thymus: pearls and pitfalls. Radiographics 30 (2):413-428 